\title{
Author Index Vol. 154, 1995
}

Augsburger, H.R. 135

Baltadjiev, G. 181

Bershadsky, A.D. 46

Calvo,A. 186

Cheong,E. 300

Chung, I.H. 162

Chung, M.S. 162

Craven, AJ. 283

Crossin, K.L. 21

Cruz-Orive, L.M. 135

Delannet, M. 63

Duband,J.L. 63

Ferguson, M.W.J. 7

Ferrari, C.S. 205

Fraher,J. 300

Friess, A.E. 196

Gabius,H.-J. 272

Gallego-Huidobro, J. 186

Garratt,A.N. 34

Geiger, B. 46

Girol,A.R. 267

Gomes Pessanha, M. 261

Hartley, L. 243

Hay,E.D. 8

Herring, S.W. 205

Hewitt, C.C. 111

Horn, R. 186

Humphries, MJ. 34

Ishikawa, K. 143

Kang,H.S. 162

Kim,HJ. 162

Koepp,H. 272

Krahn,V. 272

Krstic, R. 232

Le Minor, J.M. 236

Mandarim-de-Lacerda, C.A. 261

Markwald, R.R. 111

Maurer, JJ. 216

Mayer, B. 99 
Michiels, I. 272 Miething,A. 99 Miyata, H. 147 Monier, F. 63 Montenegro, M.A. 128 Newgreen, D.F. 7,63,243 Nicolas, D. 232 Nixon, A.J. 283 Oliani,S.M. 267 Ono,K. 143 Oomori,Y. 143 O’Rahilly,R. 167 Pallares,J. 186 Parry, A.L. 283 Pastor, L.M. 186 Paulsen, A. 169 Pearson, A.J. 283 Prakash,Y.S. 147 Prieto,A.L. 21 Rojas,M.A. 128 Rotz,A. von 196 Satoh,Y. 143 Schieber, M.H. 216 Schmidt, W. 272 Segretain, D. 224 Sieck,GC. 147 Singer, M.A. 216 Sinning, A.R. 111 Smith, R.L. 267 Spooner,B.S. 169 Stofft,E. 272 Twest, J.S. van 169 Viebahn, C. 79, 99 Wilson, D.B. 120 Wyatt,D.P. 120 Yehuda-Levenberg, S. 46 Zhan,W.Z. 147 Zschäbitz, A. 272 